\title{
Darstellungen und Vorstellungen und ihre Bedeutung für eine wirksame Metakognition beim Problemlösen und Begründen
}

\author{
JOHANN SJUTS
}

Abstract. Metacognition has one of the highest effect sizes concerning successful learning. However metacognitive activities during task solving and problem solving are not directly obvious. But they can appear by writing someone's thoughts down. The following analysis, which focusses on the level of argumentation as well as on the way of derivation, shows that the quality of representation is an essential condition for the possibility of metacognition.

Key words and phrases: metacognition, external and internal representations, competencies, educational standards, problem solving, argumentation.

ZDM Subject Classification: C30, D40, D50.

\section{Metakognition beim Mathematiklernen}

Bildung ist in der heutigen Wissensgesellschaft von allerhöchstem Rang. Mehr und mehr gewinnt auch die Bildungsforschung an Wichtigkeit. Insbesondere rückt die Wirksamkeit von Schule und Unterricht ins Augenmerk. Dabei ist vor allem von Interesse, welche Effektstärken verschiedene Einflussfaktoren auf erfolgreiches Lernen besitzen. Nach den vorliegenden Forschungserkenntnissen kommt Metakognition eine der höchsten Effektstärken zu (Wang et al. 1993, Hattie 2008).

Der Begriff Metakognition (engl. metacognition) geht auf Flavell (1976) zurück. Zunächst untersuchte die Psychologie die Frage, inwieweit Menschen ihre 
eigenen kognitiven Aktivitäten kennen und damit auch kontrollieren und steuern, inwieweit sie also über eigenes Metawissen und eigene Metastrategien verfügen.

Indes gab es zur Metakognition schon in der Mathematik eine überaus bedeutsame Vorgeschichte, ohne dass dabei der Begriff Metakognition auftrat. Überlegungen darüber, wie sich das eigene Denken und Nachdenken ins Bewusstsein heben lässt, finden sich nämlich bereits in den Werken Pólyas zum mathematischen Problemlösen. Hier ist das berühmt gewordene Buch "How to Solve It" (Pólya 1945) zu nennen. Es war dann vor allem Schoenfeld (1985, 1992), der die Ideen für die Mathematikdidaktik aufgenommen und weitergeführt hat.

Die Forschung über Metakognition ist in verschiedenen Disziplinen durch unterschiedliche Schwerpunkte bestimmt. Die Psychologie widmet sich vornehmlich der Frage, welche Rolle Metakognition beim selbstregulierten Lernen (Boekarts 1996, 1999) und beim Leseverstehen spielt. In der Mathematikdidaktik steht Problemlösen im Mittelpunkt (Schoenfeld 1985, 1992, De Corte et al. 2000, Kramarski \& Mevarech 2003, Mevarech \& Kramarski 2014).

Mittlerweile haben sich die auf Metakognition gerichteten bildungswissenschaftlichen und fachdidaktischen Fragestellungen ausgedehnt. Und auch in den staatlichen Curricula findet der Begriff Metakognition Erwähnung, ohne jedoch genau expliziert, spezifiziert oder operationalisiert zu werden.

Eine besondere kognitionstheoretische Ausrichtung der Mathematikdidaktik (in Deutschland) besteht an der Universität Osnabrück. Die dort in der Arbeitsgruppe "Kognitive Mathematik" seit vielen Jahren betriebenen Untersuchungen befassen sich mit kognitiven Prozessen und metakognitiven Aktivitäten der Lernenden und gehen dabei Fragen nach, die einerseits die mathematikdidaktische Forschung, andererseits die Gestaltung von Mathematikunterricht betreffen. Gerade diese Schwerpunktsetzung lässt die Auffassung von Mathematikdidaktik als "design science" (Wittmann 1992) zum Tragen kommen.

Metakognition beim Mathematiklernen erhält damit eine exponierte Stellung. Die in der Arbeitsgruppe "Kognitive Mathematik" auf Forschung und Entwicklung gerichtete Beschäftigung mit Metakognition ist dezidiert fachdidaktisch angelegt und durch die Besonderheit der kognitionstheoretischen Orientierung geprägt. Es geht um mathematische Lehr-Lern-Prozesse sowie um mathematische Denk- und Verstehensprozesse. Dabei werden sowohl Grundlagenforschung und Unterrichtsentwicklung, also die analytische und die konstruktive Ebene, als auch Lernpsychologie, Allgemeindidaktik und Mathematikdidaktik, also die allgemeinpädagogische und die fachdidaktische Ebene, verknüpft (Cohors-Fresenborg et al. 2010). 
Die akzentuierte Verknüpfung hat einen besonderen Niederschlag gefunden in dem Projekt "Mathematik Gut Unterrichten. Lernprozesse forschungsbezogen organisieren. Ein Qualitätsnetzwerk für Mathematiklehrkräfte", das durch die Deutsche Telekom Stiftung gefördert worden ist. Es gab zwei Arbeitsbereiche für die beteiligten Mathematiklehrkräfte (Kaune \& Cohors-Fresenborg 2010). Der eine betraf die Analyse von Lernprozessen, Lernäußerungen und Lernprodukten der Schülerinnen und Schüler, die nach theoretischen Erkenntnissen erfolgte. Das besondere Augenmerk galt der Individualität von Vorstellungen und Fehlvorstellungen (Sjuts 2007a, 2008a). Der andere umfasste Planung, Dokumentation und Auswertung von Unterrichtsprozessen. Die Methode war die videobasierte Analyse nach einem erprobten Kategoriensystem. Hier standen die auf Diskursivität und Metakognition zielenden Unterrichtsgespräche im Mittelpunkt (Kaune \& CohorsFresenborg 2010).

Ebenso zu nennen sind zwei von der Deutschen Forschungsgemeinschaft geförderte Projekte, nämlich das Projekt "Analyse von Unterrichtssituationen zur Einübung von Reflexion und Metakognition im gymnasialen Mathematikunterricht der S I" (Cohors-Fresenborg \& Kaune 2007) und das Projekt "Entwicklung eines schulfachübergreifenden Instruments zur videobasierten Klassifikation metakognitiv-diskursiver Unterrichtsqualität am Beispiel der Fächer Geschichte, Mathematik, Religion" (Cohors-Fresenborg 2012).

Der vorliegende Beitrag ist der Analyse von dokumentierten Schülerlernleistungen zuzuordnen. Dabei ist zu fragen, welche Erkenntnisse aus dieser Analyse zu gewinnen sind. Einerseits könnte daraus resultieren, passende Förder- und Unterstützungsmaßnahmen zu entwickeln, anderseits könnte es eine Konsequenz sein, den Unterricht schon im Vorfeld anders anzulegen. Möglicherweise wären vorgelagerte Maßnahmen sogar wichtiger und wirkungsvoller als nachgelagerte. Damit gerät auch die Ausbildung von Lehramtsstudierenden und angehenden Lehrkräften in den Blick. Ziel ist die theoretisch und empirisch fundierte Entwicklung von Professionalität für Schule und Unterricht.

\subsection{Metakognition}

Metakognition wird gemeinhin verstanden als das Wissen und die Kontrolle über das eigene kognitive System (Sjuts 2003, Mevarech \& Kramarski 2014). Mit dem Denken über das eigene Denken sowie der Steuerung des eigenen Denkens hat Metakognition folglich eine deklarative und eine prozedurale (exekutive) Komponente. Hinzukommen muss die Aufmerksamkeit für entsprechende Erfahrungen und Willensleistungen, also eine motivationale (sensitive) Komponente. Verkürzt 
soll damit von deklarativer, prozeduraler und motivationaler Metakognition die Rede sein.

Die deklarative Metakognition beinhaltet das Wissen eines Menschen über eigene kognitive Gegebenheiten und Funktionen (Hasselhorn 2006). Metawissen bezieht sich auf Personen-, Aufgaben- und Strategievariablen. Es umfasst somit zum einen das diagnostische Wissen, das jemand über das eigene Denken besitzt, zum anderen das bewertende Wissen über Aufgaben und Anforderungen und zum weiteren das strategische Wissen über Lösungswege und Erfolgsaussichten. Das Metawissen und damit die Entwicklung des so genannten Metagedächtnisses spielen eine Rolle bei Lern- und Gedächtnisleistungen, also bei Anforderungen des Einprägens, Behaltens und Erinnerns. Im günstigsten Fall sind einem Menschen die eigenen Stärken und Schwächen bekannt. Urteilsvermögen dagegen ist gefragt bei der Einschätzung der Schwierigkeiten, die eine gestellte Aufgabe enthält. Eine Bewertung bezieht sich selbstverständlich auf die Aufgabe selbst, vor allem aber auf das Verhältnis zwischen eigenem Leistungsstand und Aufgabenschwierigkeit. Von Bedeutung ist zudem ein Wissen über Strategien, darüber, ob sich Lösungsideen zur Bewältigung einer Aufgabe eignen, darüber, wie wirkungsvoll bestimmte Vorgehensweisen sind.

Die prozedurale Metakognition verbindet die vor, während und nach einer Aufgabenbearbeitung liegenden Tätigkeiten des Planens, Überwachens und Überprüfens, bei denen eine Person sich gewissermaßen selbst über die Schulter blickt. Laut zu denken oder das eigene Denken und Handeln zu protokollieren, sind Regulation und Kontrolle unterstützende Methoden. Eine Wirkung von Vorausschau, Innehalten und Rückschau kann schließlich darin liegen, die eigene Position zu überdenken und gegebenenfalls zu verändern.

Voraussetzung dafür, dass jemand Metakognition betreibt, sind Bereitschaft und Gespür (Hasselhorn 2006). Es müssen somit Motivation und Willenskraft für den Einsatz metakognitiver Strategien vorliegen. Oder sie müssen entwickelt werden, etwa dadurch, dass die Lösung einer gestellten Aufgabe als Herausforderung empfunden wird. Ebenso ist ein Gespür für das Leistungsvermögen eigener kognitiver Aktivitäten unerlässlich. Eine solche Sensibilität ist Folge metakognitiver Erfahrungen. Denn aus diesen Erfahrungen resultieren Fähigkeiten zur Introspektion und zur Einschätzung einer zweckmäßigen Nutzung des eigenen deklarativen und prozeduralen Metawissens.

Im tatsächlichen Vollzug von Metakognition gibt es ein kompliziertes Zusammenspiel der angesprochenen Komponenten, die sich empirisch kaum trennen 
lassen. Gleichwohl beschäftigt sich die Forschung mit einer Vielfalt von Fragestellungen, die sich bestimmten Teilbereichen widmen (Mevarech \& Kramarski 2014).

\subsection{Darstellungen und Vorstellungen}

Beim Mathematiklernen sind Darstellungen und Vorstellungen von zentraler Bedeutung. Darstellungen sind externe Repräsentationen (Schwank 2003) in sprachlicher oder nicht-sprachlicher Form. Bei Darstellungen geht es um die Frage: Wie schreibt man etwas auf? Vorstellungen sind interne Repräsentationen von nicht unmittelbarer Zugänglichkeit. Bei Vorstellungen geht es um die Frage: Wie legt man sich etwas im Kopf zurecht? (Sjuts 2014)

Wenn von Vorstellungen hier die Rede ist, sind die so genannten Grundvorstellungen (Blum et al. 2004) eingeschlossen. Grundvorstellungen beschreiben, auf welche - durchaus verschiedenen, aber bedeutungsadäquaten und tragfähigen - Weisen man einen mathematischen Begriff oder eine mathematische Operation auffassen kann. Bei Modellvorstellungen handelt es sich um leistungsfähige mentale Modelle, die als organisierende Stützstrukturen des Denkens und Verstehens fungieren. Anders als Grundvorstellungen und Modellvorstellungen umfassen Vorstellungen in der Bezeichnung Schülervorstellungen ein ganzes Spektrum. Dieses reicht - bezogen auf die Begriffs- oder Operationsbedeutung - von einem inadäquaten bis zu einem adäquaten Zurechtlegen im Kopf.

\subsection{Analyse von Metakognition im Mathematikunterricht}

Metakognition im Unterricht ist nicht per se wirksam. Es kommt darauf an, Unterrichtsgespräche so zu arrangieren und zu moderieren, dass metakognitive und diskursive Aktivitäten in merkmalspezifischer Weise zum Ausdruck kommen. Um entsprechende Merkmale zu identifizieren und zu klassifizieren, eignet sich das von Cohors-Fresenborg und Kaune (2007, 2010) entwickelte Kategoriensystem, und zwar vor allem für solche Unterrichtsgespräche, in denen von der Sache her ein argumentatives Vorgehen geboten ist.

Gegenstand der Klassifikation sind beobachtbare Lehr-Lern-Aktivitäten. Zugrunde liegen dafür Unterrichtstranskripte und manchmal als Ergänzung Tafelund Folienbilder. Das Kategoriensystem enthält vier Kategorien, neben den drei Kategorien zur Metakognition, nämlich Planung, Monitoring und Reflexion, noch die Kategorie Diskursivität (Kaune \& Cohors-Fresenborg 2010). Bei der Planung 
geht es um die Organisation des jeweils weiteren Vorgehens in dem Unterrichtsgespräch. Zum Monitoring gehören die Kontrolle der Lehr-Lern-Prozesse und die jeweilige Selbstüberwachung. Die Reflexion umfasst alle Aktivitäten, die die Vorkenntnisse, das jeweils zuvor Geschehene und die (Zwischen-)Ergebnisse betreffen. Insgesamt wird die prozedurale Metakognition folglich in vielfältiger Weise erfasst.

In einem engen Zusammenhang zur Metakognition steht Diskursivität (Nowińska 2016). Es geht dabei um die Einhaltung von Gesprächsregeln und die Nachvollziehbarkeit der Argumentationen (Kaune \& Cohors-Fresenborg 2010). Im Unterricht zeigt sich Diskursivität darin, dass die am Gespräch Beteiligten ihre Aussagen und Argumente präzise formulieren und ihre Beiträge durch Bezugspunkte und -personen verankern. Sie haben vorhergehende oder folgende Äußerungen zu verorten und durch Bezugnahme und Vergewisserung schließlich für Klarheit im Denken und Gründlichkeit im Verstehen zu sorgen. Erwähnenswert ist noch die Unterkategorie der negativen Diskursivität, zu der alle Beeinträchtigungen der Diskursqualität gehören (Kaune \& Cohors-Fresenborg 2010).

In komprimierter Form werden die klassifizierten Lehr-Lern-Aktivitäten auf einem Kategorienstrahl (Nowińska 2016) abgetragen. So ist es möglich, eine längere Unterrichtsgesprächssequenz mit einem Blick zu analysieren und die Anteile der metakognitiven und diskursiven Aktivitäten auf Lehrer- und Schülerseite direkt abzulesen.

An mehreren Stellen im Kategoriensystem tritt die Bedeutung von Darstellungen und Vorstellungen hervor (Kaune \& Cohors-Fresenborg 2010). Bei der Planung steht die Frage im Vordergrund, welche Darstellung (Formel, Grafik, Term, Text) besonders geeignet ist, Einsicht in das gewählte Vorgehen zu erzielen. $\mathrm{Zu}$ bedenken ist auch, auf welche Weise Fehlvorstellungen ins Auge fallen können. Beim Monitoring sind insbesondere die Terminologie und die Notation zu überwachen. Die kontinuierliche Kontrolle soll sicherstellen, dass eigene und fremde Fehlvorstellungen aufgedeckt werden. Bei der Reflexion gilt es, die Adäquatheit der Darstellungen zu prüfen sowie das Zusammenspiel von Darstellungen und Vorstellungen einer Analyse zu unterziehen (Kaune \& Cohors-Fresenborg 2010).

Bisherige Untersuchungen belegen die Nützlichkeit des Kategoriensystems. Und sie geben Hinweise darauf, in welcher Art und Weise metakognitive und diskursive Aktivitäten zu einem erfolgreichen Unterricht beitragen. Als ein wesentliches Ergebnis kann festgehalten werden, dass die durch Präzision und Stringenz geprägte Diskursivität für Metakognition in Unterrichtsgesprächen essentiell ist (Nowińska 2016), dass es folglich als passend erscheint, die entsprechende Unterrichtskultur als metakognitiv-diskursiv zu kennzeichnen. 
Es existieren zahlreiche allgemein- und fachdidaktische Vorarbeiten. Die empirische Unterrichtsforschung hat dokumentierten und transkribierten Unterricht auf verschiedene Weisen zu analysieren versucht. Dabei galt das Augenmerk den fachübergreifend feststellbaren Argumentationen (Toulmin 1975, Walter 2005, Budke et al. 2015) oder (im Fach Mathematik) den Interaktionen (Voigt 1984). Im Vordergrund standen Analyse und Interpretation. Für die (Weiter-)Entwicklung beruflicher Professionalität ist indes die Verknüpfung theoretischer, empirischer und praktischer Komponenten ausschlaggebend. "Nicht das Betrachten des Unterrichtsvideos an sich macht einen zur besseren Lehrperson, sondern (...) das Herbeiziehen von theoretischen Erkenntnissen erweitert das Denken und Wissen über Unterricht und lässt das tägliche Unterrichtsgeschäft unter veränderter und erweiterter Perspektive planen, durchführen und evaluieren." (Krammer \& Reusser 2004, S. 98 f.)

\subsection{Analyse von Metakognition bei Aufgabenbearbeitungen in Mathematik}

Beim Bearbeiten von Aufgaben und beim Lösen von Problemen sind metakognitive Aktivitäten nicht unmittelbar ersichtlich. Sie lassen sich indes durch Verschriftlichen von Gedanken nach außen kehren. Gerade die schriftlich fixierte Überwachung und Kommentierung des eigenen Denkens und Handelns erweist sich bei alltäglichen Aufgabenbearbeitungen als gewinnbringend (Sjuts 2001, 2002a).

Bereits die Aufgabenstellung kann Metakognition anregen. Lehrerinnen und Lehrer stehen damit vor folgenden Fragen: Wie gestaltet man Aufgaben, die Denkprozesse aufdecken und Verstehensprozesse fördern? Wie regt man metakognitive Aktivitäten an? Dazu liegen Vorschläge zur Aufgabenstellung vor, die ausdrücklich zum Explizieren, Variieren, Formalisieren (Cohors-Fresenborg, Sommer \& Sjuts 2004), Analysieren, Synthetisieren auffordern und darauf abzielen, dass Lernende bei der Aufgabenbearbeitung in bewusster Weise agieren (Sjuts 2008c).

Explizieren (Erläutern - Verschriftlichen - Begründen): Erläutern kann Unsichtbares sichtbar werden lassen. ("Erläutere dein Vorgehen.") Verschriftlichen macht Handeln und Denken bewusst. ("Schreibe auf, wie du rechnest.") Begründen präzisiert den Gedankengang. ("Begründe deine Antwort.")

Variieren (Notieren - Zeichnen - Übertragen): Variieren der Darstellung deckt das Denken auf. ("Fertige eine zeichnerische Darstellung an und beschrifte sie.") Aufgaben mit mehreren Lösungswegen lassen individuelles Denken zum Ausdruck 
kommen. Übertragen in eine andere Darstellungsform kann die Lösungswahrscheinlichkeit erhöhen.

Formalisieren (Symbolisieren - Formulieren - Repräsentieren): Formalisieren unterstützt mathematisches Denken. Formalisieren reduziert Komplexität und schafft Durchschaubarkeit (Cohors-Fresenborg, Sommer \& Sjuts 2004). Formulieren verdeutlicht das Erfassen und Vorstellen formaler Ausdrücke. Die Auseinandersetzung mit Wissensrepräsentationen (Schreibweisen, Äußerungen) ist zentral für metakognitive Aktivitäten.

Analysieren (Vergleichen - Kommentieren - Überprüfen): Erörterndes Vergleichen von Aufgabenbearbeitungen mit verschiedenen Darstellungen und Ergebnissen klärt auch das eigene Denken. Analysieren und Kommentieren von Fehlvorstellungen oder Dialogen anderer dienen der Selbstförderung. Zweckmäßig sind Aufgaben zur Selbstüberprüfung der eigenen Überlegungen. ("Du wirst gefragt, wie du die Richtigkeit sicherstellst. Wie antwortest du?")

Synthetisieren (Ergänzen - Fortsetzen - Produzieren): Ergänzungen, Fortsetzungen und Zusammensetzungen (vorgegebener Lösungsansätze) stimulieren das eigene Denken. Das Finden unterschiedlicher Lösungswege ist eine wirkungsvolle Form des Festigens. Das Produzieren gleichartiger Aufgaben ist Ausdruck eines verständnisintensiven Lernens.

$\mathrm{Zu}$ konstatieren ist indes, dass eine Aufgabenerweiterung um explizite Aufforderungen zur Metakognition nicht etabliert ist. Eine solche Gestaltung von Aufgaben oder Teilaufgaben ist in Schulbüchern zur Mathematik nur sehr selten zu finden. Auch in Schulleistungsstudien, bei Vergleichsarbeiten oder Zentralarbeiten ist keine nennenswerte Berücksichtigung festzustellen.

\section{Metakognition beim Problemlösen und Begründen}

Seit mehreren Jahren gelten Bildungsstandards im Fach Mathematik für den Primarbereich (KMK 2004). Damit rücken allgemeine und inhaltsbezogene mathematische Kompetenzen, die für das Mathematiklernen und die Mathematik insgesamt charakteristisch sind, in den Vordergrund. Kinder sollen Gelegenheiten erhalten, selbst Probleme zu lösen und über Mathematik zu kommunizieren. Das Ziel ist die Entwicklung eines gesicherten Verständnisses mathematischer Inhalte. Die in den Bildungsstandards beschriebenen allgemeinen Kompetenzen sind Problemlösen, Kommunizieren, Argumentieren, Modellieren und Darstellen von Mathematik. Mittels passender Aufgaben lässt sich der Ausprägungsgrad einer jeden 
Kompetenz feststellen und auf diese Weise die Anschlussfähigkeit beim Übergang von der Grundschule auf die weiterführenden Schulen zum Ausdruck bringen.

Die Kompetenzen Problemlösen und Argumentieren lassen sich für Schülerinnen und Schüler am Ende der 4. Jahrgangsstufe wie folgt konkretisieren (KMK 2004):

Problemlösen bedeutet, mathematische Kenntnisse, Fertigkeiten und Fähigkeiten bei der Bearbeitung problemhaltiger Aufgaben anzuwenden, Lösungsstrategien zu entwickeln und zu nutzen (z.B. systematisch zu probieren), Zusammenhänge zu erkennen, zu nutzen und auf ähnliche Sachverhalte zu übertragen.

Argumentieren bedeutet, mathematische Aussagen zu hinterfragen und auf Korrektheit zu prüfen, mathematische Zusammenhänge zu erkennen und Vermutungen zu entwickeln, Begründungen zu suchen und nachzuvollziehen.

Die folgende Analyse stellt Metakognition beim Problemlösen und Begründen in den Mittelpunkt. Dabei ist bei einer schwierig zu lösenden Aufgabe von einem Problem die Rede, wenn vertraute Lösungsroutinen nicht vorliegen, die Anforderung, das Problem zu bewältigen, also darin besteht, einen neuen Gedankengang zu entwickeln.

Nach Pólya (1945) sind folgende Schritte für ein erfolgreiches Problemlösen geeignet: Zunächst gilt es, das Problem zu verstehen und die darin enthaltene Aufgabe zu erfassen. Danach sind Ideen für eine Strategie zu entwickeln. Sodann ist der ausgedachte Plan auszuführen und begleitend zu kontrollieren. Schließlich ist eine Rückschau zu halten, also zu prüfen, ob alles stimmt oder irgendetwas verbessert werden muss.

Gerade die beiden letzten Schritte nehmen das Argumentieren und in einem engeren Sinn das Begründen in den Blick. Zu kontrollieren und zu prüfen ist die Stringenz des Vorgehens und die Lückenlosigkeit.

Die nachstehende Analyse geht folgenden Untersuchungsfragen nach: Welche Schritte des Problemlösens sind rekonstruierbar? Welche metakognitiven Aktivitäten sind identifizierbar? Von welcher Art sind die Herleitungen? Von welchem Grad sind die Begründungen?

Für die qualitative Analyse von Aufgabenbearbeitungen liegen Verschriftlichungen vor. Der Analyse liegt die Annahme zugrunde, dass ihnen die Stufen des Problemverstehens und der Strategieentwicklung nicht unmittelbar zu entnehmen, die der Ausführung und der Rückschau dagegen wohl zu entnehmen sind, dass außerdem die Darstellungen der Aufgabenbearbeitungen auf die Vorstellungen von einer Begründung oder von einer für vollständig gehaltenen Begründung schließen lassen. 


\subsection{Aufgabe}

Die folgende Aufgabe ist eine leicht abgewandelte Fassung der Aufgabe 530523 aus der Regionalrunde der Mathematik-Olympiade 2013/2014 im Schuljahrgang 5 (Sjuts 2014). (Die dokumentierten Aufgabenbearbeitungen stammen von talentierten und interessierten Schülerinnen und Schülern einer Arbeitsgemeinschaft aus dem Schuljahrgang 5.)

Aufgabe "Personen im Abteil"

Über die Personen im Abteil eines Zuges ist bekannt:

(1) Die Anzahl aller Personen im Abteil ist eine Quadratzahl zwischen 26 und 50.

(2) Es ist ein Erwachsener mehr als Kinder.

(3) Es sind zweimal so viele Mädchen wie Jungen.

(4) Es sind drei Frauen mehr als Männer.

a) Aus der Aussage (1) kann man herleiten, dass für die Anzahl der Personen nur zwei Zahlen in Frage kommen. Welche Zahlen sind das? Begründe.

b) Aus der Aussage (2) kann man dann herleiten, wie viele Personen, wie viele Erwachsene und wie viele Kinder im Abteil sind. Gib diese Anzahlen an und begründe.

c) Wie viele Mädchen und wie viele Jungen befinden sich im Abteil? Begründe.

d) Wie viele Frauen und wie viele Männer sind es? Begründe.

Hinweis: Eine Quadratzahl ist eine Zahl, die als Ergebnis einer Multiplikation einer Zahl mit sich selbst entsteht. Zum Beispiel sind 81 und 144 Quadratzahlen, denn $81=9 \cdot 9$ und $144=12 \cdot 12$.

\subsection{Erste Teilaufgabe}

Dazu seien einige Aufgabenbearbeitungen nun näher betrachtet. Neben dem Ergebnis in Zahlen sind vor allem die Begründungen von Bedeutung. Sie können einen unterschiedlichen Grad aufweisen.

Der geringste Grad besteht in der Angabe der beiden Zahlen 36 und 49 ohne weitere Erklärungen, Begründungen oder Erläuterungen - wie bei Robert.

Robert:

$$
\text { a) } 36,49
$$


Möglicherweise ist diese Antwort für ihn von hoher Evidenz. In der Mathematik pflegt man gelegentlich zu sagen, dass man das Ergebnis "unmittelbar sieht", dass es also "offensichtlich" ist.

Einen höheren Grad hat die Version, die neben der Angabe der beiden Zahlen den Nachweis enthält, dass es sich um Quadratzahlen handelt. Dies zeigt die folgende Aufgabenbearbeitung von Nina.

Nina:

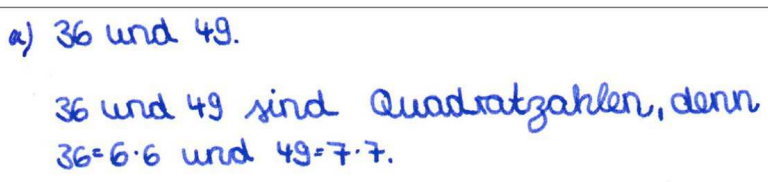

Über diesen Grad von Begründung hinaus geht die Aufgabenbearbeitung, in der außerdem angemerkt wird, dass die beiden Quadratzahlen zwischen 26 und 50 liegen (Vera).

Vera:

$$
\begin{aligned}
& \text { a.) In der tuss age steht, dass zahlen } \\
& \text { zwischen } 26 \text { und } 50 \text { rauskommen } \\
& \text { missen. } \\
& 6.6=36 \\
& 7.7=49 \\
& 36 \text { und } 49 \text { lieg en zurishen } 26 \text { und } \\
& 50 \text { und sind Quadratzahlen. }
\end{aligned}
$$

Und noch weiter reicht die Lösung, in der zusätzlich erwähnt wird, dass es nur diese und keine weiteren Quadratzahlen zwischen 26 und 50 gibt (Cora).

Cora:

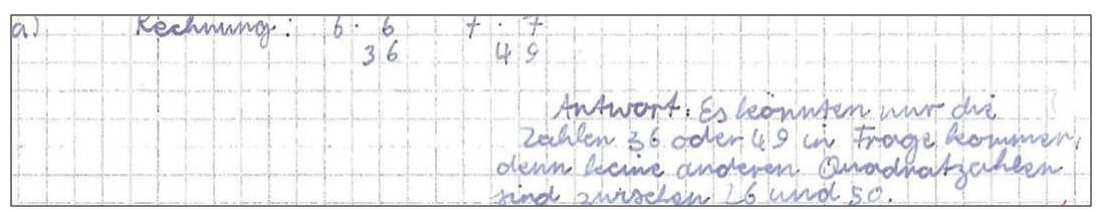


Die Aufgabenbearbeitung mit dem höchsten Grad enthält auch dazu noch eine Begründung (Sandra).

Sandra:

$\begin{array}{ll}\text { a) } 1 \cdot 1=1 & \text { Antwort: } \\ 2 \cdot 2=2 & \text { Es sind die Quddratzahlen } 36 \text { und } \\ 3 \cdot 3=9 & 49 \text { gemeint, weil die anderen Quadrat- } \\ 4 \cdot 4=16 & \text { zahten zu klein oder zu gropsind. } \\ 5 \cdot 5=25 & \\ 6 \cdot 6=36 & \\ 7 \cdot 7=49 & \\ 8 \cdot 8=64 & \\ 9 \cdot 9=81 & \end{array}$

Zusammenstellung:

\begin{tabular}{|l|l|l|l|l|l|}
\hline & $\begin{array}{l}\text { Teilaufgabe } \\
\text { a) }\end{array}$ & $\begin{array}{l}\text { Teilaufgabe } \\
\text { b) }\end{array}$ & $\begin{array}{l}\text { Teilaufgabe } \\
\text { b) }\end{array}$ & $\begin{array}{l}\text { Teilaufgabe } \\
\text { c) }\end{array}$ & $\begin{array}{l}\text { Teilaufgabe } \\
\text { d) }\end{array}$ \\
\hline & $\begin{array}{l}\text { Grad der } \\
\text { Begründung }\end{array}$ & $\begin{array}{l}\text { Grad der } \\
\text { Begründung }\end{array}$ & $\begin{array}{l}\text { Art der } \\
\text { Herleitung }\end{array}$ & $\begin{array}{l}\text { Art der } \\
\text { Herleitung }\end{array}$ & $\begin{array}{l}\text { Art der } \\
\text { Herleitung }\end{array}$ \\
\hline Robert &.$/$. & & & & \\
\hline Nina & gering & & & & \\
\hline Vera & mittel & & & & \\
\hline Cora & hoch & & & & \\
\hline Sandra & hoch & & & & \\
\hline David & & & & & \\
\hline Felix & & & & & \\
\hline Simon & & & & & \\
\hline Axel & & & & & \\
\hline Lena & & & & & \\
\hline
\end{tabular}

Es ist durchaus denkbar, dass Robert eine vollständige Begründung im Kopf vorgenommen hat. Er schreibt allerdings nur das Ergebnis seiner Überlegungen auf. Damit ist eine Überprüfung des eigenen Denkens erschwert. Je ausführlicher die Überlegungen verschriftlicht werden, desto leichter fällt die Kontrolle. Auch die Struktur der Verschriftlichung ist von unterschiedlicher Eignung für eine Überprüfung. Diese sich steigernden Möglichkeiten einer wirksamen Metakognition verdeutlichen die Lösungen von Nina über Vera und Cora bis Sandra.

Resümieren lässt sich dies: Die Darstellungen der Aufgabenbearbeitungen lassen auf die dahinter liegenden Vorstellungen schließen. Die Qualität der Darstellung (der Wissensrepräsentation überhaupt) ist eine wesentliche Bedingung 
der Möglichkeit von Metakognition. Und es ist plausibel, dass mit der Qualität der Darstellung die Wirksamkeit von Metakognition steigt.

Die dokumentierten Aufgabenbearbeitungen stammen von Schülerinnen und Schülern aus dem Schuljahrgang 5. Die Aufgabenbearbeitungen werfen Fragen auf: Ist es Ziel des Mathematikunterrichts im Laufe der Jahre zuvor gewesen, eine Modellvorstellung von Begründung zu entwickeln? Haben diese Schülerinnen und Schüler im Mathematikunterricht eine Modellvorstellung von Begründung aufgebaut?

Die Bildungsstandards im Fach Mathematik für den Primarbereich (KMK 2004) weisen Argumentieren als eigene prozessbezogene mathematische Kompetenz aus. Begründungen zu suchen und nachzuvollziehen, wird explizit genannt. In Verbindung mit anderen prozessbezogenen Kompetenzen, die in diesem $\mathrm{Zu}$ sammenhang bedeutsam sind, wie Problemlösen, Kommunizieren und Darstellen, wird man annehmen dürfen, dass im Grundschulunterricht hinreichend Gelegenheit war, eine tragfähige Vorstellung von einer Begründung aufzubauen.

In den Bildungsstandards im Fach Mathematik für den Primarbereich heißt es ausdrücklich, dass im Primarbereich "die Grundlage für das Mathematiklernen in den weiterführenden Schulen und für die lebenslange Auseinandersetzung mit mathematischen Anforderungen des täglichen Lebens geschaffen" (KMK 2004) wird. "Die Standards beschreiben die inhaltlichen und allgemeinen mathematischen Kompetenzen, die Kinder am Ende der Grundschulzeit erworben haben sollen. Erwartet wird, dass die Schülerinnen und Schüler diese Kompetenzen in außermathematischen ("Anwendungsorientierung") und in innermathematischen ("Strukturorientierung") Kontexten nutzen können." (KMK 2004)

Bei Aufgabenstellungen der Mathematik-Olympiade findet sich stets der Hinweis: "Der Lösungsweg mit Begründungen und Nebenrechnungen soll deutlich erkennbar sein. Du musst also auch erklären, wie du zu Ergebnissen und Teilergebnissen gelangt bist. Stelle deinen Lösungsweg logisch korrekt und in grammatisch einwandfreien Sätzen dar." Vielleicht ist es ratsam, zumindest in den letzten Jahren der Grundschule eine solche Anforderung durchgängig zu stellen, um den vorgeschriebenen Bildungsstandards gerecht zu werden.

\subsection{Zweite Teilaufgabe}

Auch dazu seien Aufgabenbearbeitungen genauer in den Blick genommen. Dabei soll neben dem Grad der Begründung auch die Art der Herleitung betrachtet werden. 
Der geringste Grad der Begründung liegt in der ausschließlichen Angabe der Zahlen (49 Personen, 25 Erwachsene, 24 Kinder). Der nächsthöhere Grad enthält zudem einen Nachweis (Gesamtzahl, Unterschied). Weiterreichend ist eine Herleitung mit zugehörigen Erläuterungen, die die Gesamtzahl 49 betrifft (David).

David:

$$
\begin{aligned}
& \text { b) Es sind } 49 \text { Personen im } \\
& \text { Wagen. Es sind } 25 \text { Erwachsene } \\
& \text { Und } 24 \text { Kinder. Das liegt daran, } \\
& \text { dass es eime gerade und } \\
& \text { eine ungerade Anzahl von } \\
& \text { Leuten im Wagen seim muss. }
\end{aligned}
$$

An diesem Beispiel wird eine Art der Herleitung deutlich. Sie ist geprägt durch die Sicht auf Beziehungsstrukturen und Eigenschaften, hier auf Summanden mit dem Unterschied 1 und auf die Eigenschaften von Zahlen, gerade oder ungerade zu sein. Ist nämlich die Anzahl der Erwachsenen um 1 größer als die der Kinder, ist die eine Anzahl gerade, die andere ungerade. Die Summe aus einer geraden Zahl und einer ungeraden Zahl ist eine ungerade Zahl. Somit ist $49(=25+24)$ die Gesamtzahl (David).

Zu diesem Ergebnis kommt Felix auch. Er zeigt zudem, dass die Zahl 36 nicht in Frage kommen kann.

Felix:

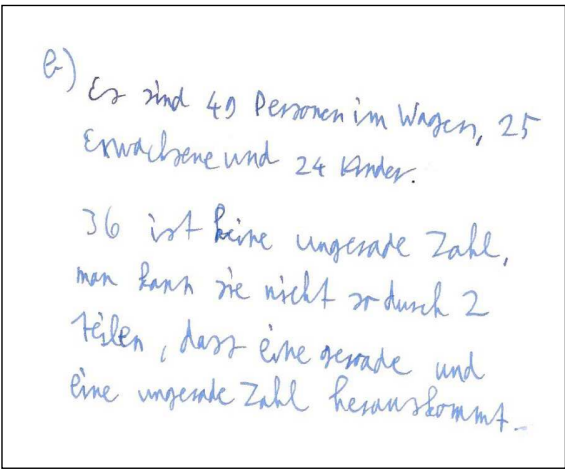

Hier ist eine andere Art der Herleitung erkennbar. Sie ist durch eine Prozesshaftigkeit geprägt. Das Zerlegen einer Zahl in zwei Summanden ist ein Prozess, 
auch wenn dafür unpassende Formulierungen benutzt werden. So schreibt Felix: "36 ist keine ungerade Zahl, man kann sie nicht so durch 2 teilen, dass eine gerade und eine ungerade Zahl herauskommt." Durch 2 zu teilen, meint hier, die Zahl in zwei Summanden zu zerlegen. Die Gesamtzahl in zwei Summanden zu zerlegen, kann auch probeweise begonnen und dann versuchsweise oder schrittweise verändert werden, bis es (bei der Gesamtzahl 49) aufgeht oder man feststellt, dass es (bei der Gesamtzahl 36) nicht aufgehen kann (Simon).

Simon:

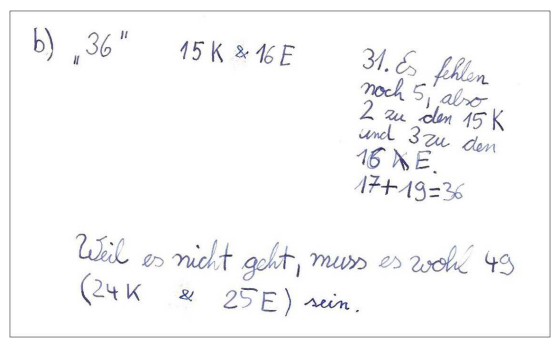

Zusammenstellung:

\begin{tabular}{|l|l|l|l|l|l|}
\hline & $\begin{array}{l}\text { Teilaufgabe } \\
\text { a) }\end{array}$ & $\begin{array}{l}\text { Teilaufgabe } \\
\text { b) }\end{array}$ & $\begin{array}{l}\text { Teilaufgabe } \\
\text { b) }\end{array}$ & $\begin{array}{l}\text { Teilaufgabe } \\
\text { c) }\end{array}$ & $\begin{array}{l}\text { Teilaufgabe } \\
\text { d) }\end{array}$ \\
\hline & $\begin{array}{l}\text { Grad der } \\
\text { Begründung }\end{array}$ & $\begin{array}{l}\text { Grad der } \\
\text { Begründung }\end{array}$ & $\begin{array}{l}\text { Art der } \\
\text { Herleitung }\end{array}$ & $\begin{array}{l}\text { Art der } \\
\text { Herleitung }\end{array}$ & $\begin{array}{l}\text { Art der } \\
\text { Herleitung }\end{array}$ \\
\hline Robert &.$/$ & & & & \\
\hline Nina & gering & & & & \\
\hline Vera & mittel & & & & \\
\hline Cora & hoch & & & & \\
\hline Sandra & hoch & & & & \\
\hline David & & mittel & prädikativ & & \\
\hline Felix & & hoch & funktional & & \\
\hline Simon & & hoch & funktional & & \\
\hline Axel & & & & & \\
\hline Lena & & & & & \\
\hline
\end{tabular}

Die kognitionstheoretisch orientierte Mathematikdidaktik hat Unterschiede in Strukturen und Strategien mathematischen Denkens ausfindig gemacht (Schwank 2003). Strukturen und Strategien lassen sich bei bestimmten Aufgabenstellungen typisieren (Sjuts 2002b, 2007b, 2008b) und - auch bei hier vorliegenden Aufgabenbearbeitungen - identifizieren. Die Darstellung lässt Rückschlüsse auf eine 
Präferenz in der kognitiven Struktur zu, die die Art und Weise des Zurechtlegens von in Aufgabenstellungen gegebenen Situationen bestimmt. Man unterscheidet prädikativ-logisches Zurechtlegen und funktional-logisches Zurechtlegen. Prädikatives Denken lässt sich an der Beziehungsstrukturiertheit, funktionales Denken an der Prozesshaftigkeit von Vorstellungen erkennen.

Welche Bedeutung haben diese Unterschiede für die Metakognition? Eine Stärke der prozesshaften Herleitung, eines Vorgehens mit einem gedanklichen Hineinversetzen, liegt darin, dass die Plausibilität, vor allem jedoch die NichtPlausibilität eines Ergebnisses auffällt. Eine gewisse metakognitive Aktivität ist dieser Art also inhärent. Anders ist es bei einer eigenschaftsbezogenen Herleitung. Hier ist eine Darstellung nützlich, wenn nicht sogar erforderlich, an der die Überwachung des Denkens erfolgt.

\subsection{Dritte Teilaufgabe}

Die beiden Arten der Herleitung finden sich auch in den Aufgabenbearbeitungen zur Teilaufgabe c).

Auf die unterschiedlichen Grade der Begründung wird nun nicht mehr näher eingegangen. Die Vorgehensweisen, eine gegebene Zahl in zwei Summanden mit bestimmten Bedingungen zu zerlegen, sollen dagegen erläutert werden.

Zunächst werden die Aufgabenbearbeitungen zur Anzahl der Mädchen und zur Anzahl der Jungen betrachtet. Hier spielt das Verhältnis der Zahlen eine Rolle.

Eine Aufgabenbearbeitung (Sandra) zeichnet sich durch die Nutzung der Eigenschaft der Beziehung der gesuchten Zahlen zueinander aus. Wenn zweimal so viele Mädchen wie Jungen im Abteil sind, dann handelt es sich um zwei Anteile an Mädchen und um einen Anteil an Jungen, also um insgesamt drei Anteile an Kindern. Hier ist die Herleitung auch konsequent formalisiert, wie das schon in der ersten Teilaufgabe festzustellen war.

Sandra:

$$
\text { c) } \begin{aligned}
& 24: 3=8 \\
& 8 \cdot 2=16 \\
& 8 \cdot 1=8
\end{aligned}
$$

Eine ebenfalls präzise Herleitung stammt von David. 
David:

$$
\begin{aligned}
& \text { c) (-1) } 49 \\
& \text { (2) } 25 \text { Erwachsene, } 24 \text { kinder } \\
& \text { (3) } 24: 3=8 \quad 8+8=16 \\
& 8 \text { Jungen } 16 \text { Mädchen }
\end{aligned}
$$

In den folgenden Aufgabenbearbeitungen wird probeweise vorgegangen: Hier werden die Startzahlen teils begründet, teils versuchsweise gewählt.

Felix:

$$
\begin{aligned}
& \text { c) } 24: 2=12 \\
& 12 \text { Jmoten } 12 \text { Mid-hen } \\
& 8 \text { Imngen } 16 \text { widiken } \\
& \text { Es and } 16 \text { withen und } 8 \text { ansen, } \\
& \text { da kie Midclen 2-mal ro viel } \\
& \text { aind whe die Juagen. }
\end{aligned}
$$

Simon:

$$
\begin{aligned}
& \text { d) Es sind } 24 \text { Yiinder. } \\
& 5 \mathrm{~J} \text { \& } 10 \mathrm{M} \text { klappt niht, es fehlen noch } 9 . \\
& 8 \mathrm{~J} \text { \& } 16 \mathrm{M} \text { klappt, abo ist es richtig. }
\end{aligned}
$$

Robert:

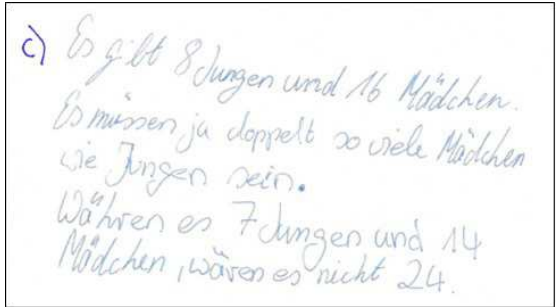


Mit einem zu Felix' Vorgehen ähnlich aussehenden Ansatz scheitert Axel. Er rechnet $24: 2=12$ und $12: 2=6$ und $12+6=18$. Die so berechneten Zahlen 18 und 6 sind aber falsch.

Axel:

$$
\begin{aligned}
& \text { c) Wan muss zweimval so viele Nädchen wire Junos } \\
& \text { halen, also muss mon } 24: 2=12 \text { und } 17: 2=6 \text { und } \\
& 12+6=18 \text { rechnen, also sind es } 18 \text { Nödichen und } 6 \text { Jurvojen. }
\end{aligned}
$$

Zusammenstellung:

\begin{tabular}{|l|l|l|l|l|l|}
\hline & $\begin{array}{l}\text { Teilaufgabe } \\
\text { a) }\end{array}$ & $\begin{array}{l}\text { Teilaufgabe } \\
\text { b) }\end{array}$ & $\begin{array}{l}\text { Teilaufgabe } \\
\text { b) }\end{array}$ & $\begin{array}{l}\text { Teilaufgabe } \\
\text { c) }\end{array}$ & $\begin{array}{l}\text { Teilaufgabe } \\
\text { d) }\end{array}$ \\
\hline & $\begin{array}{l}\text { Grad der } \\
\text { Begründung }\end{array}$ & $\begin{array}{l}\text { Grad der } \\
\text { Begründung }\end{array}$ & $\begin{array}{l}\text { Art der } \\
\text { Herleitung }\end{array}$ & $\begin{array}{l}\text { Art der } \\
\text { Herleitung }\end{array}$ & $\begin{array}{l}\text { Art der } \\
\text { Herleitung }\end{array}$ \\
\hline & & & & & \\
\hline Robert &.$/$ & & & funktional & \\
\hline Nina & gering & & & & \\
\hline Vera & mittel & & & & \\
\hline Cora & hoch & & & prädikativ & \\
\hline Sandra & hoch & & prädikativ & prädikativ & \\
\hline David & & mittel & funktional & funktional & \\
\hline Felix & & hoch & funktional & funktional & \\
\hline Simon & & hoch & & funktional & \\
\hline Axel & & & & & \\
\hline Lena & & & & & \\
\hline
\end{tabular}

\subsection{Vierte Teilaufgabe}

Bei der Teilaufgabe d) ist die Differenz der Zahlen vorgegeben. Indes finden sich bei den Aufgabenbearbeitungen zur Herleitung der Anzahl der Frauen und der Anzahl der Männer erneut die genannten Arten.

In der nachstehenden Lösung (von Sandra) wird der Lösungsgang präzise dargestellt. Dabei wird gleich zu Beginn die Anzahldifferenz benutzt, um eine Anschlussrechnung durchzuführen, die nach einer weiteren Rechnung zum Ergebnis führt. 
Sandra:

$$
\text { d) } \begin{aligned}
25-3 & =22 \\
22: 2 & =11 \\
11+3 & =14
\end{aligned}
$$

In anderen Aufgabenbearbeitungen wird probeweise vorgegangen: Erneut werden die Startzahlen teils begründet, teils versuchsweise gewählt. Danach gilt es, die Zahlen zu variieren und zu "gucken, wie es passt" (Lena).

In einigen Fällen ist die Summe der gewählten Startwerte 25. Die Summe bleibt dann konstant, während die Anzahlwerte in unterschiedlichen Sprüngen verändert werden (Felix, Axel, Lena). In anderen Fällen bleibt der Abstand zunächst versuchsweise gewählter Anzahlwerte konstant, während die Summe sich mit der Änderung der Anzahlwerte ändert, bis das Ergebnis 25 erreicht ist (Simon, Robert).

Felix:

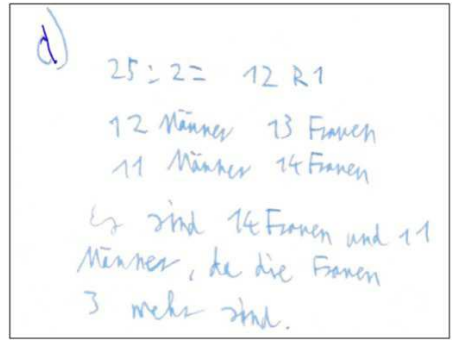

Simon:

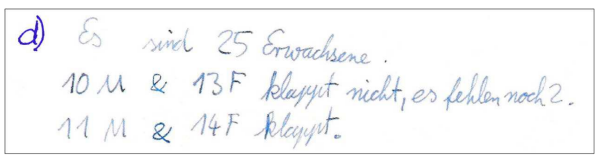

Axel:

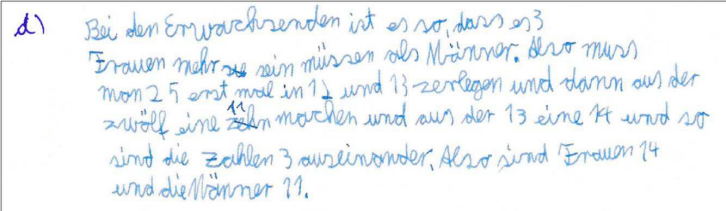


Robert:

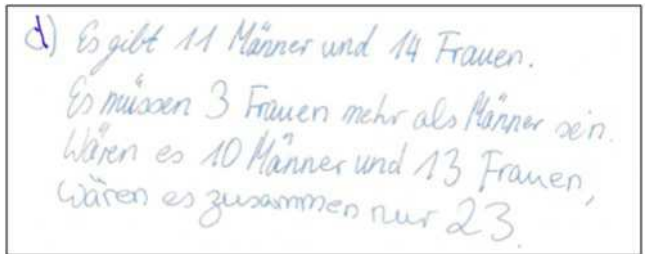

Lena:

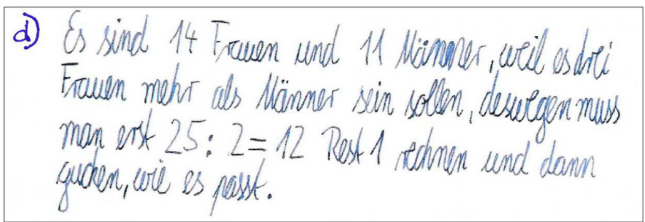

Zusammenstellung:

\begin{tabular}{|l|l|l|l|l|l|}
\hline & $\begin{array}{l}\text { Teilaufgabe } \\
\text { a) }\end{array}$ & $\begin{array}{l}\text { Teilaufgabe } \\
\text { b) }\end{array}$ & $\begin{array}{l}\text { Teilaufgabe } \\
\text { b) }\end{array}$ & $\begin{array}{l}\text { Teilaufgabe } \\
\text { c) }\end{array}$ & $\begin{array}{l}\text { Teilaufgabe } \\
\text { d) }\end{array}$ \\
\hline & $\begin{array}{l}\text { Grad der } \\
\text { Begründung }\end{array}$ & $\begin{array}{l}\text { Grad der } \\
\text { Begründung }\end{array}$ & $\begin{array}{l}\text { Art der } \\
\text { Herleitung }\end{array}$ & $\begin{array}{l}\text { Art der } \\
\text { Herleitung }\end{array}$ & $\begin{array}{l}\text { Art der } \\
\text { Herleitung }\end{array}$ \\
\hline Robert &.$/$ & & & & \\
\hline Nina & gering & & & funktional & funktional \\
\hline Vera & mittel & & & & \\
\hline Cora & hoch & & & & \\
\hline Sandra & hoch & & & prädikativ & prädikativ \\
\hline David & & mittel & prädikativ & prädikativ & \\
\hline Felix & & hoch & funktional & funktional & funktional \\
\hline Simon & & hoch & funktional & funktional & funktional \\
\hline Axel & & & & funktional & funktional \\
\hline Lena & & & & & funktional \\
\hline
\end{tabular}

Das Zerlegen einer Zahl in zwei Summanden mit gegebener Differenz und das Zerlegen einer Zahl in zwei Summanden mit gegebenem Verhältnis treten in zwei typisierbaren Varianten mathematischen Denkens auf. In diesen wenigen Fällen zeigt sich sogar eine bemerkenswerte Stabilität. Erkennbar ist der Unterschied in den Darstellungen. Diese sind Ausdruck offenbar unterschiedlicher Vorstellungen vom Zerlegen einer Zahl in zwei Summanden unter bestimmten Bedingungen. 
Die Aufgabe ist, wie erwähnt, der Mathematik-Olympiade entnommen. Die einzelnen Aufgabenteile zu lösen, ist schon deshalb nicht eine Routineanforderung. Sie stellen für Schülerinnen und Schüler im Schuljahrgang 5 ein herausforderndes Problem dar. Auch die Individualität der Aufgabenbearbeitungen ist dafür ein deutlicher Beleg.

Ist in den Aufgabenbearbeitungen eine Schrittfolge des Problemlösens im Sinne von Pólya zu erkennen? Nur an wenigen Stellen liegt eine Formulierung über das Vorgehen oder die Strategie vor. Zumeist bleibt also verborgen, worüber sich die jeweilige Person Gedanken gemacht hat. Damit können nur aus den Verschriftlichungen, den Darstellungen, Rückschlüsse gezogen werden über die Vorstellungen.

Offensichtlich ist es in den dokumentierten Fällen gelungen, den Aufgabentext und die Aufgabenstellung zu erfassen, also eine Vorstellung zu entwickeln über den Sachverhalt und eine mögliche Herangehensweise zur Beantwortung der gestellten Frage. Eine begleitende Beobachtung und Kontrolle kann ebenfalls angenommen werden. Sie zeigt sich in manchen Formulierungen ("In der Aussage steht, dass Zahlen zwischen 26 und 50 rauskommen müssen.", "klappt, also ist es richtig", "dann gucken, wie es passt"). Den jeweiligen Darstellungen kann entnommen werden, dass Stringenz in der Gedankenführung eine hohe Bedeutung hat, um die Richtigkeit der Ergebnisse abzusichern. Es ist auch bemerkenswert, in welchem Maße vollständig ausformulierte Sätze gebildet werden. Eine Rückschau ist dagegen nicht erkennbar.

Damit sind metakognitive Aktivitäten angesprochen. Sie liegen vor allem und in erster Linie in der Genauigkeit der schriftlich fixierten Rechnungen und Argumentationen. Inwieweit ein innerer Plan am Anfang gestanden hat, ist nicht ersichtlich. In jedem Fall hat die begleitende Selbstüberwachung ihren Niederschlag in der Darstellung gefunden. Und umgekehrt erlaubt die Darstellung eine Selbstüberwachung. Es zeigt sich hier schließlich der Zusammenhang von Repräsentation und Metakognition. Metakognition ist möglich, wenn Werkzeuge von Sprache und Mathematik zur Wissensrepräsentation zur Verfügung stehen (Sjuts 2003).

\section{Wirksamkeit von Metakognition}

Aus der Analyse der Aufgabenbearbeitungen lassen sich gewisse Erkenntnisse gewinnen. Sie betreffen zunächst das Wechselspiel von Darstellungen und Vorstellungen. Bildliche, sprachliche, symbolische und formale Darstellungen sind 
externe Wissensrepräsentationen einer Person, die als Ausdruck ihrer Vorstellungen, ihrer internen Wissensrepräsentationen, gelten können. Und diese Darstellungen, die die betreffende Person anfertigt, auf die sie dabei blickt, können auf die Vorstellungen zurückwirken. Besteht die Anforderung darin, eine mathematische Aufgabe zu bearbeiten oder ein Problem zu lösen, könnte man sogar von einem förderlichen Wechselspiel sprechen.

Die eine oder die andere Art von Herleitung einzuschlagen, scheint dabei abhängig von kognitiven Dispositionen der jeweiligen Person zu sein (Schwank 2003). So kann es sein, dass die Art und Weise, in der ein Problem gestellt wird, nicht auf Resonanz in der kognitiven Struktur stößt. Es ist folglich von Interesse für das Lehren und Lernen, eine möglichst genaue Kenntnis von der Beziehung zwischen externen und internen Wissensrepräsentationen zu erhalten. Diese Beziehung ist für Lern-, Denk- und Problemlösevorgänge in folgender Weise von Bedeutung: Wenn man annimmt, dass im Lernprozess eine bestimmte externe Repräsentation (nur) eine bestimmte interne Repräsentation evoziert, dann ist nicht nur die innere Verfasstheit genau zu ergründen, sondern ebenso wichtig ist es, sich über die Wahl der äußeren Darstellung sorgfältig Gedanken zu machen (Schwank 2003).

Wenn eine Aufgabe als schwierig, gar als nahezu unlösbar erscheint und damit ein Problem darstellt, können gewisse Vorgehensweisen weiterhelfen. Lernende müssen dies wissen. Der Wechsel der äußeren Repräsentationsform ist ein wichtiges Prinzip. Der Mathematik wohnt Multimodalität als Kennzeichen inne. Der Darstellungswechsel (etwa zwischen Text, Tabelle, Symbol, Graph, Bild) ermöglicht es im günstigsten Fall, dass eine Resonanz zur kognitiven Struktur eintritt (Schwank 2003) und dass die Bewältigung einer zu lösenden Aufgabe erleichtert wird.

Sandra und Simon finden eine ihrer kognitiven Struktur je gemäße Lösung. Sandra scheint über eine Sicht auf die Gesamtsituation zu verfügen, die es ihr ermöglicht, alle relevanten Eigenschaften und Beziehungen zu erfassen und eine Lösung mit einer vollständigen Begründung zu erreichen. Die Darstellung selbst begünstigt eine Kontrolle der Lösung. Simon scheint sich in die Situation hineinzuversetzen und sie mit für die Lösung in Frage kommenden Zahlen unter den vorgegebenen Bedingungen durchzuspielen, um dabei prüfend festzustellen, dass es "nicht klappt", oder, dass, wenn es "klappt", die Zahl das gesuchte Ergebnis ist. So vollzieht er die Kontrolle des Lösungsvorgangs.

Je nach kognitiver Struktur fällt die Art der metakognitiven Aktivität also unterschiedlich aus. Und die Metakognition ist trotz der Unterschiedlichkeit 
in den Vorgehensweisen durchaus wirksam. Die Sicht auf die Situation und das Hineinversetzen in die Situation (Sjuts 2002b, 2007b) erlauben auf verschiedene, gleichwohl passende Weise eine Kontrolle der Problemlösung. Dennoch wären zu der in der Aufgabenstellung genannten Anforderung, für die Lösung eine Begründung zu liefern, eine bewusste Vorausschau und eine bewusste Rückschau, also eine überlegte Planung und eine strukturierte Überprüfung vorzunehmen, in jedem Fall eine wirksamkeitssteigernde Ergänzung.

Die Selbstregulation beim Planen, Überwachen und Prüfen ist gerade beim Problemlösen von hoher Bedeutung. Denn der Lösungsprozess ist nicht durch abrufbare Routinen von vornherein klar. Er entsteht durch planvolles oder auch versuchsweises Vorgehen, das mit einer ständigen Kontrolle verbunden ist. In ähnlicher Weise ist das aus mehreren Schritten oder komplexen Argumentationen bestehende Begründen zu überwachen.

Wie können Schülerinnen und Schüler das lernen? Musterlösungen sind geeignet, beispielhaft und modellhaft zu zeigen, welche Erwartungen hinsichtlich der Verschriftlichung zu erfüllen sind. Selbstverständlich sind durchgängig zu verlangende Überarbeitungen eigener Darstellungen ein wesentlicher Bestandteil des Lernens.

Ganz sicher bedeutsam ist der Unterricht in seiner Ausgestaltung. Und hier kommt es auf die Untrennbarkeit von Metakognition und Diskursivität an (Sjuts 2008b). Das beginnt für die Lehrperson bereits damit, die Einzel- oder Gruppenarbeitsergebnisse rasch zu registrieren, um gegebenenfalls eine Vorauswahl für die Präsentation und die Auswertung zu treffen. Denn es müssen zentrale mathematische Ideen und ebenso, wenn vorhanden, typische Sichtweisen und Fehlvorstellungen zur Darbietung, zur Erörterung und zur Klärung kommen. Und das Unterrichtsgespräch darüber, pointiert ausgedrückt: über die Wissensrepräsentationen, hat sich daran auszurichten, den Qualitätsansprüchen metakognitiver und diskursiver Aktivitäten Geltung zu verschaffen. Ein solches Vorgehen berücksichtigt einerseits ein hohes Maß an Vielfalt und Individualität, es sorgt andererseits zugleich für Verbindlichkeit und Klarheit.

Es bedarf weiterer Forschung, Metakognition hinsichtlich vielfältiger Fragestellungen zu untersuchen und tiefergehend zu erfassen. Genauer zu klären ist, wie Lernen, Denken und Verstehen mit metakognitiven und diskursiven Aktivitäten im Unterricht zusammenhängen. Mit dem so gewonnenen Wissen sind schließlich Konzepte zur Gestaltung von Lehr-Lern-Prozessen zu entwickeln, in denen Metakognition ihre Wirksamkeit entfalten kann. 


\section{Literatur}

[1] W. Blum, R. vom Hofe, A. Jordan and M. Kleine, Grundvorstellungen als aufgabenanalytisches und diagnostisches Instrument bei PISA, in: Mathematische Kompetenzen von Schülerinnen und Schülern in Deutschland: Vertiefende Analysen im Rahmen von PISA-2000, (M. Neubrand, ed.), Verlag für Sozialwissenschaften, Wiesbaden, 2004, 145-157.

[2] M. Boekaerts, Teaching Students Self-Regulated Learning: A Major Success in Applied Research, in: Contemporary Psychology in Europe, (J. Georgas et al., eds.), Hogrefe \& Huber, Seattle, 1996, 245-259.

[3] M. Boekarts, Self-Regulated Learning: Where we are today, International Journal of Educational Research 31 (1999), 445-457.

[4] Fachlich argumentieren lernen: Didaktische Forschungen zur Argumentation in den Unterrichtsfächern, (A. Budke, M. Kuckuck, M. Meyer, F. Schäbitz, K. Schlüter and G. Weiss, eds.), Waxmann, Münster, 2015.

[5] E. Cohors-Fresenborg, Metakognitive und diskursive Aktivitäten - ein intellektueller Kern im Unterricht der Mathematik und anderer geisteswissenschaftlicher Fächer, in: Formate Fachdidaktischer Forschung, Empirische Projekte - historische Analysen - theoretische Grundlegungen, (H. Bayrhuber, U. Harms, B. Muszynski, B. Ralle, M. Rothgangel, L.-H. Schön, H. J. Vollmer and H.-G. Weigand, eds.), Waxmann, Münster, 2012, 145-162.

[6] E. Cohors-Fresenborg, N. Sommer and J. Sjuts, Komplexität von Denkvorgängen und Formalisierung von Wissen, in: Mathematische Kompetenzen von Schülerinnen und Schülern in Deutschland, Vertiefende Analysen im Rahmen von PISA 2000, (M. Neubrand, ed.), VS Verlag, Wiesbaden, 2004, 109-144.

[7] E. Cohors-Fresenborg and C. Kaune, Modelling Classroom Discussions and Categorising Discursive and Metacognitive Activities, in: Proceedings of CERME 5, 2007, 1180-1189.

[8] E. Cohors-Fresenborg, S. Kramer, F. Pundsack, J. Sjuts and N. Sommer, The role of metacognitive monitoring in explaining differences in mathematics achievement, ZDM Mathematics Education 42, DOI 10.1007/s11858-010-0237-x (2010), 231-244.

[9] E. De Corte, L. Verschaffel and P. Op't Eynde, Self-regulation: A characteristic and a goal of mathematics education, in: Self-regulation: Theory, research, and applications, (P. Pintrich, M. Boekaerts and M. Zeidner, eds.), Lawrence Erlbaum Associates, Mahwah, NJ, 2000, 687-726.

[10] J. Flavell, Metacognitive aspects of problem solving, in: The nature of intelligence, (L. Resnik, ed.), Hillsdale, NJ, Lawrence Erlbaum, 1976, 231-236.

[11] M. Hasselhorn, Metakognition, in: Handwörterbuch Pädagogische Psychologie, 3. Aufl., (D. H. Rost, ed.), Weinheim, 2006, 480-485.

[12] J. Hattie, Visible Learning, Routledge, London, 2008.

[13] Mathematik Gut Unterrichten, Analyse von Mathematikunterricht bezüglich metakognitiver und diskursiver Aktivitäten, (C. Kaune and E. Cohors-Fresenborg, eds.), Osnabrück, 2010. 
[14] KMK, Bildungsstandards im Fach Mathematik für den Primarbereich (Jahrgangsstufe 4), Beschluss der Kultusministerkonferenz vom 15. Oktober 2004., 2004.

[15] B. Kramarski and Z. Mevarech, Enhancing mathematical reasoning in the classroom: The effects of cooperative learning and metacognitive training, American Educational Research Journal 40, no. 1 (2003), 281-310.

[16] K. Krammer and K. Reusser, Unterrichtsvideos als Medium der Lehrerinnen- und Lehrerbildung, in: Seminar - Lehrerbildung und Schule, Vol. 10, no. 4, 2004, 80-101.

[17] Z. Mevarech and B. Kramarski, Critical Maths for Innovative Societies: The Role of Metacognitive Pedagogies, OECD Publishing, 2014, http://dx.doi.org/10.1787/9789264223561-en.

[18] E. Nowińska, Leitfragen zur Analyse und Beurteilung metakognitiv-diskursiver Unterrichtsqualität, Osnabrück, 2016.

[19] G. Pólya, How to Solve It, Princeton University Press, Princeton, 1945.

[20] A. H. Schoenfeld, Metacognitive and epistemological issues in mathematical understanding, in: Teaching and learning mathematical problem solving: Multiple research perspectives, (E. A. Silver, ed.), Erlbaum, Hillsdale, NJ, 1985, 361-380.

[21] A. H. Schoenfeld, Learning to think mathematically: Problem solving, metacognition, and sense making in mathematics, in: Handbook of research on mathematics teaching and learning, (D. A. Grouws, ed.), Macmillan, New York, 1992, 334-370.

[22] I. Schwank, Einführung in prädikatives und funktionales Denken, Zentralblatt für Didaktik der Mathematik 35, no. 3 (2003), 70-78.

[23] J. Sjuts, Metakognition beim Mathematiklernen: das Denken über das Denken als Hilfe zur Selbsthilfe, Der Mathematikunterricht 47, no. 1 (2001), 61-68.

[24] J. Sjuts, Metacognition in Mathematics Lessons, in: Developments in Mathematics Education in German-speaking Countries, Selected Papers from the Annual Conference on Didactics of Mathematics, Bern 1999, (H.-G. Weigand et al., eds.), Hildesheim, Berlin, 2002a, 76-87.

[25] J. Sjuts, Unterschiedliche mentale Konstruktionen beim Aufgabenlösen, Eine Fallstudie zur Mathematik als Wissensrepräsentation, Journal für Mathematik-Didaktik 23, no. 2 (2002b), 106-128.

[26] J. Sjuts, Metakognition per didaktisch-sozialem Vertrag, Journal für MathematikDidaktik 24, no. 1 (2003), 18-40.

[27] J. Sjuts, Mini-Forschung im Berufsfeld Schule, Steigerung von Unterrichtsqualität und Verbesserung von Lehrerausbildung, dargestellt am Beispiel des Grundschulprojekts "Metakognition beim mathematischen Denken", Leer, 2007a.

[28] J. Sjuts, Kompetenzdiagnostik im Lernprozess - auf theoriegeleitete Aufgabengestaltung und -auswertung kommt es an, mathematica didactica 30, no. 2 (2007b), $33-52$.

[29] J. Sjuts, Diagnostik in Mathematik, Aufbau diagnostischer Kompetenz durch Mini-Forschung zur Metakognition beim mathematischen Denken, Leer, 2008a. 
[30] J. Sjuts, Kognitionsanalysen als Bedingung der Möglichkeit von Prozessdiagnostik in Echtzeit, in: Algebraisches Denken, Festschrift für Lisa Hefendehl-Hebeker, (B. Barzel, T. Berlin, D. Bertalan and A. Fischer, eds.), Hildesheim, 2008b, 123-135.

[31] J. Sjuts, Aufgaben diagnostisch gestalten, Denkprozesse aufdecken und Verstehen fördern, mathematik lehren 150 (2008c), 58-61.

[32] J. Sjuts, Vorstellungen und Darstellungen: Evidenzbasierte Diagnostik und Gestaltung mathematischer Lehr-Lern-Prozesse, Beiträge zum Mathematikunterricht (2014), 1139-1142.

[33] S. Toulmin, Der Gebrauch von Argumenten, Scriptor, Kronberg, 1975.

[34] J. Voigt, Interaktionsmuster und Routinen im Mathematikunterricht, Theoretische Grundlagen und mikroethnographische Falluntersuchungen, Beltz, Weinheim, 1984.

[35] P. Walter, Welchen Sinn macht die Allgemeine Didaktik? Zur Theorie und Empirie unterrichtlicher Interaktionen, in: Mathematikdidaktik im Wissenschaftsgefüge: Zum Verstehen und Unterrichten mathematischen Denkens, Vol. 2, Festschrift für Elmar Cohors-Fresenborg, (C. Kaune, I. Schwank and J. Sjuts, eds.), Osnabrück, 2005, 195-207.

[36] M. C. Wang, G. D. Haertel and H. J. Walberg, Toward a knowledge base for school learning, Review of Educational Research 63, no. 3 (1993), 249-294.

[37] E. C. Wittmann, Mathematikdidaktik als "design science", Journal für Mathematik-Didaktik 13, no. 1 (1992), 55-70.

JOHANN SJUTS

UNIVERSITÄT OSNABRÜCK

DEUTSCHLAND

E-mail: jsjuts@uni-osnabrueck.de

(Received June, 2016) 